\title{
Performance Evaluation of Hybrid Precoded Millimeter Wave Wireless Communication System on Color Image Transmission
}

\author{
Sk. Shifatul Islam ${ }^{1}$, Shammi Farhana Islam², Mahmudul Haque Kafi ${ }^{1}$, Shaikh Enayet Ullah ${ }^{1}$ \\ ${ }^{1}$ Department of Applied Physics and Electronic Engineering, University of Rajshahi, Rajshahi, Bangladesh \\ ${ }^{2}$ Department of Material Science and Engineering, University of Rajshahi, Rajshahi, Bangladesh
}

Email address:

chisty56@gmail.com (Sk. S. Islam),shammi.farhana@gmail.com (S. F. Islam), mahmudkafi49@gmail.com (M. H. Kafi), enayet_apee@ru.ac.bd (S. E. Ullah)

\section{To cite this article:}

Sk. Shifatul Islam, Shammi Farhana Islam, Mahmudul Haque Kafi, Shaikh Enayet Ullah. Performance Evaluation of Hybrid Precoded Millimeter Wave Wireless Communication System on Color Image Transmission. International Journal of Wireless Communications and Mobile Computing. Vol. 4, No. 2, 2016, pp. 37-45. doi: 10.11648/j.wcmc.20160402.15

Received: April 10, 2016; Accepted: April 22, 2016; Published: May 12, 2016

\begin{abstract}
In this paper, a comprehensive study has been on the suitability of implementation of hybrid precoding scheme in performance analysis of the future generation wireless communication system. The 256-by-32 multi antenna supported simulated system incorporates various types of modern and classical channel coding schemes such as Low density parity check (LDPC), Repeat and Accumulate (RA), 1/2-rated convolutional and non-binary Bose-Chadhuri-Hocquenghem (BCH) and ZeroForcing (ZF) signal detection technique. With consideration of ray path geometry based mmWave MIMO fading channel and properly designed precoders and combiners and their applicability in simulation works, it is seen from computer simulation results that the presently considered simulated system outperforms in retrieving color image in LDPC channel coding and 16QAM digital modulation and Zero-Forcing (ZF) signal detection schemes.
\end{abstract}

Keywords: Hybrid Precoding, Channel Coding, ZF, mmWave and Bit Error Rate (BER)

\section{Introduction}

The millimeter wave (mmWave) with frequency spectrum band ranging from $30 \mathrm{GHzto} 300 \mathrm{GHz}$ is expected to be a key component in the next generation $5 \mathrm{G}$ wireless communication systems. It enables the extensive use of allocated frequency spectrum to support greater data traffic for various multimedia services such as broadband mobile and backhaul services. The mmWave spectrum holds tremendous potential for providing multi-Gigabits-persecond data rates in upcoming cellular systems. One of the fundamental goals for $5 \mathrm{G}$ wireless mobile networks is to increase data rates through extreme densification of base stations with massive multiple-input-multiple-output (MIMO). In mmWave frequency bands, it has become a challenging task to execute cellular communication properly due to blockage, absorption, diffraction and penetration of mmWaves. However, advancement of CMOS radiofrequency technology along with the very small wavelength of mmWave signals allows for the packing of large-scale antenna arrays at both the transmit and receive ends and thus provides highly directional beam forming gains with reduced interference and acceptable signal-to-noise ratio (SNR) [1, 2]. In perspective of considering the potential of using of millimeter wave (mmWave) frequency for future $5 \mathrm{G}$ wireless cellular communication systems, an emphasis is being given on the study of large-scale antenna arrays for achieving highly directional beamforming. The conventional fully digital beamforming methods which require one radio frequency $(\mathrm{RF})$ chain per antenna element is not viable for large-scale antenna arrays due to the high cost and high power consumption of RF chain components in high frequencies. To address the challenge of these hardware constraints, a hybrid beamforming architecture can be considered in which the overall beamformer consists of a low-dimensional digital beamformer followed by an RF beamformer implemented using analog phase shifters [3].

It is known from literature reviewing that Samsung 
Electronics, an industry leader in exploring mmWave bands for mobile communications, has tested a technology that can achieve 2 Gbps data rate with $1 \mathrm{~km}$ range in an urban environment. Furthermore, Professor Theodore Rappaport and his research team at the Polytechnic Institute of New York University have demonstrated that mobile communications at $28 \mathrm{GHz}$ in a dense urban environment such as Manhattan, NY, is feasible with a cell size of $200 \mathrm{~m}$ using two $25 \mathrm{dBi}$ antennas, one at the BS and the other at the $\mathrm{UE}$, which is readily achievable using array antennas and the beamforming technique [4].

The present study has been confined on the performance evaluative study of the simulated large-scale antenna mmWave system under consideration of hybrid beamforming structures.

\section{Signal Processing Techniques}

In this section, various signal processing techniques used for channel coding, geometry based fading channel estimation, hybrid precoding designing and signal detection have been outlined below.

\subsection{Bose-Chadhuri-Hocquenghem (BCH) Channel Coding}

$\mathrm{BCH}$ codes are a class of cyclic codes discovered in 1959 by Hocquenghem and independently in 1960 by Bose and Ray-Chaudhuri. The BCH codes are both binary and multi level The binary $\mathrm{BCH}$ code is parameterized by an integer $\mathrm{m} \geq 3$. The $\mathrm{t}$ error correcting $\mathrm{BCH}$ code of length $\mathrm{n}$ is of varying nature depending on the value of $\mathrm{m}$ and is given by $\mathrm{n}=2^{\mathrm{m}}-1$. Its roots include $2 \mathrm{t}$ consecutive powers of $\alpha$, the primitive element of $\mathrm{GF}\left(2^{\mathrm{m}}\right)$ [5]. In our study, a binary $\mathrm{BCH}$ code is of length 127 with a message is of length 64 have been used. In such $[127,64] \mathrm{BCH}$ code, the value of errorcorrection capability, $\mathrm{t}$ is 10 .

\subsection{Low Density Parity-Check Matrix (LDPC) Channel Coding}

LDPC code is a linear error correction code. Its parity check matrix $\mathrm{H}$ used in this paper is of $64 \times 128$ sized and this matrix is sparse containing less non zero elements irregularly in each row and column. The number of non zero element in each column ranges from 1 to 3 and the number of non zero element in each row ranges from 5 to 6 . The $1 / 2 .-$ rated irregular LDPC code used here has a code length of 128bits.The parity-check matrix His formed from a concatenation of two matrices $\mathrm{A}$ and $\mathrm{P}$, each with a dimension of $64 \times 64)$. The columns of the parity-check matrix $\mathrm{H}$ is rearranged to produce a modified form of parity-check matrix $\overline{\mathrm{H}}$. With rearranged matrix elements, the matrix $\mathrm{A}$ becomes non-singular and it is further processed to undergo LU decomposition. The parity bits sequence $p$ is considered to have been produced from a block based input binary data sequence $\mathrm{u}=\left[\mathrm{u}_{1} \mathrm{u}_{2} \mathrm{u}_{3} \mathrm{u}_{4} \ldots \ldots . \mathrm{u}_{64}\right]^{\mathrm{T}}$ and three matrices $\mathrm{L}, \mathrm{U}$ and $\mathrm{P}($ of $\overline{\mathrm{H}})$ using the following Matlab notation:

$$
\mathrm{p}=\bmod (\mathrm{U} \backslash(\mathrm{L} \backslash \mathrm{z}), 2) ; \text { where }, \mathrm{z}=\bmod \left(\mathrm{P}^{*} \mathrm{u}, 2\right) ;
$$

The LDPC encoded $128 \times 1$ sized block based binary data sequencec is formulated from concatenation of parity check bit $\mathrm{p}$ and information bit $\mathrm{u}$ as: $[\mathrm{c}]=[\mathrm{p} ; \mathrm{u}]$, The first 64 bits of the codeword matrix [c]are the parity bits and the last 64bitsarethe information bits . The Log Domain Sum-Product LDPC is a soft decision decoding algorithm operating alternatively on the bit nodes and the check nodes through the Tanner graph. In such scheme, the received bits $(0 / 1)$ are primarily converted into $-1 /+1$ and assumed to be corrupted with AWGN channel noise of variance $\sigma^{2}(=\mathrm{N} 0 / 2), \mathrm{N} 0$ is the noise power spectral density. In processing, various required parameter values are compute diteratively with a view to finding out the mostly acceptable code words that satisfies the conditionc $\overline{\mathrm{H}}^{\mathrm{T}}=0[6,7]$.

\subsection{Repeat and Accumulate Channel Coding}

The RA is a powerful modern error-correcting channel coding scheme. In such scheme, all the extracted binary bits from the color image has been arranged into a single block and the binary bits of the such block is repeated 2 times and rearranged into a single block containing binary data which is double of the number of input binary data[8].

\subsection{Convolutional Channel Coding}

In Convolutional Channel Coding, Convolutional codes are commonly specified by three parameters $(\mathrm{n}, \mathrm{k}, \mathrm{m}): \mathrm{n}=$ number of output bits; $\mathrm{k}=$ number of input bits; $\mathrm{m}=$ number of memory registers. The quantity $\mathrm{k} / \mathrm{n}$ called the code rate and it is a measure of the efficiency of the code.

The constraint length $\mathrm{L}(=\mathrm{k}(\mathrm{m}-1))$ represents the number of bits in the encoder memory that affect the generation of the $n$ output bits. Our presently considered Convolutional Channel scheme is specified with a coding rate of $1 / 2$ and a constraint length of 7 . The code generator polynomials G1 and G2are171 and 133 in octal numbering system and can be written as [9]:

$$
\begin{aligned}
& \mathrm{G} 1=\mathrm{x}^{0}+\mathrm{x}^{2}+\mathrm{x}^{3}+\mathrm{x}^{5}+\mathrm{x}^{6}=10101101011=133 \\
& \mathrm{G} 2=\mathrm{x}^{0}+\mathrm{x}^{1}+\mathrm{x}^{2}+\mathrm{x}^{3}+\mathrm{x}^{6}=11111001=171
\end{aligned}
$$

\subsection{Zero-Forcing (ZF) Signal Detection}

In the 256 x 32 MIMO hybrid precoded system, the transmitted and received signals are represented by $\mathrm{X}=\left[\mathrm{X}_{1}\right.$, $\left.\mathrm{X}_{2} \ldots \ldots \ldots \mathrm{X}_{256}\right]^{\mathrm{T}}$ and $\mathrm{Y}=\left[\mathrm{Y}_{1}, \mathrm{Y}_{2} \ldots \ldots . \mathrm{Y}_{32}\right]^{\mathrm{T}}$ respectively. If $\mathrm{N}=$ $\left[\mathrm{N}_{1}, \mathrm{~N}_{2} \ldots \ldots \mathrm{N}_{32}\right]^{\mathrm{T}}$ denotes the white Gaussian noise with a variance $\sigma_{\mathrm{n}}{ }^{2}$ and the channel matrix is represented by $\mathrm{H}=\left[\mathrm{H}_{1} \mathrm{H}_{2} \ldots . . \mathrm{H}_{256}\right]$, we can write

$$
Y=\mathbf{H} X+N
$$

As the interference signals from other transmitting antennas are minimized to detect the desired signal, the detected desired signal from the transmitting antenna with inverting channel effect by a weight matrix $\mathrm{W}$ is given by

$$
\tilde{X}=\left[\tilde{X}_{1}, \tilde{X}_{2}, \ldots . \tilde{X}_{256}\right]^{T}=W Y
$$


In Zero-Forcing (ZF) signal detection scheme, the ZF weight matrix is given by

$$
\mathrm{W}_{\mathrm{ZF}}=\left(\mathrm{H}^{\mathrm{H}} \mathrm{H}\right)^{-1} \mathrm{H}^{\mathrm{H}}
$$

and the detected desired signal from the transmitting antenna is given by [10]

$$
\tilde{X}_{\mathrm{ZF}}=\mathrm{W}_{\mathrm{ZF}} \mathrm{Y}
$$

\subsection{MIMO Fading Channel Estimation}

In estimation of ray path geometry based $32 \times 256$ sized mmWave MIMO fading channel $\mathrm{H}$, it is assumed that the $\mathrm{N}_{\mathrm{t}}(=256)$ transmitting and $\mathrm{N}_{\mathrm{r}}(=32)$ receiving antenna sare arranged in uniform linear array (ULA).Such MIMO channel has limited scattering with $\mathrm{Lu}(=6)$ scatterers. Each scatterer is assumed to contribute a single propagation path between the base station (BS) and mobile station(MS). The geometrical channel model $\mathrm{H} \in \mathrm{C}^{\mathrm{N} \times \mathrm{N}}{ }_{t}$ can be written as:

$$
\mathrm{H}=\sqrt{\frac{\mathrm{N}_{\mathrm{t}} \mathrm{N}_{\mathrm{r}}}{\mathrm{L}_{\mathrm{u}}} \sum_{\mathrm{l}=1}^{\mathrm{L}_{\mathrm{u}}} \alpha_{\mathrm{u}, 1} \mathrm{a}_{\mathrm{MS}}\left(\theta_{\mathrm{u}, 1}\right) \mathrm{a}_{\mathrm{BS}}^{*}\left(\varphi_{\mathrm{u}, 1}\right)}
$$

where, $\alpha_{\mathrm{u}, \mathrm{l}}$ is the complex gain of the lth path including the path loss. The variable $\theta_{\mathrm{u}, 1}$ and $\varphi_{\mathrm{u}, 1} \in[0,2 \pi]$ are the lth path's angle of arrival and departure(AoAS/AoDs) respectively. Finally, ${ }_{B S}\left(\varphi_{u, 1}\right)$ and $a_{M S}\left(\theta_{u, 1}\right)$ are the antenna array response vectors of the $\mathrm{BS}$ and $\mathrm{MS}$ respectively.

With available knowledge of the geometry of uniform linear antenna arrays, ${ }^{\mathrm{a}} \mathrm{BS}\left(\varphi_{\mathrm{u}, 1}\right)$ is defined as:

$$
\mathrm{a}_{\mathrm{BS}}\left(\varphi_{\mathrm{u}, 1}\right)=\frac{1}{\sqrt{\mathrm{N}_{\mathrm{t}}}}\left[1, \mathrm{e}^{\mathrm{j} \frac{2 \pi}{\lambda} d \sin \left(\varphi_{\mathrm{u}, 1}\right), \ldots \ldots \ldots \ldots . . . \mathrm{j}\left(\mathrm{N}_{\mathrm{t}}-1\right) \frac{2 \pi}{\lambda} d \sin \left(\varphi_{\mathrm{u}, 1}\right)}\right]^{\mathrm{T}}
$$

And

$$
a_{M S}\left(\theta_{u, 1}\right)=\frac{1}{\sqrt{N_{r}}}\left[1, e^{j \frac{2 \pi}{\lambda} d \sin \left(\theta_{u, 1}\right), \ldots \ldots \ldots \ldots . . .} e^{j\left(N_{\mathrm{r}}-1\right) \frac{2 \pi}{\lambda} d \sin \left(\theta_{u, 1}\right)}\right]^{T}
$$

where, $\lambda$ is the signal wavelength and $d$ is the distance between two consecutive antenna elements.

The MIMO channel $\mathrm{H}$ is further normalized to get its Frobenius norm value $[11,12]$

$$
\mathrm{E}\left[\left\|\mathrm{H}_{\mathrm{F}}^{2}\right\|\right]=\mathrm{N}_{\mathrm{t}} \mathrm{N}_{\mathrm{r}}
$$

\subsection{Precoder and Combiner Designing}

The optimal unconstrained precoder $\mathrm{F}^{*}$ and optimal unconstrained combiner $\mathrm{W}^{*}$ can be estimated from implementation of singular value decomposition (SVD) to MIMO channel $\mathrm{H}$ in normalized form. The unconstrained RF precoder $\mathrm{F}_{\mathrm{RF}}$ at the transmitter side controls phases of the up converted RF signal. Its each $(i, j)$ th element is given by

$$
F_{R F}(i, j)=\frac{1}{\sqrt{\mathrm{N}_{t}}} e^{j \varphi_{i, j}}
$$

Where $\varphi_{i, j}$ is the unquantized phase of $(i, j)$ th element of unconstrained $\mathrm{RF}$ precoder $\mathrm{F}_{\mathrm{RF}}$ Each entry of $\mathrm{F}_{\mathrm{RF}}$ are quantized up to $\mathrm{B}$ bits of precision, each quantized to its nearest neighbor based on closest Euclidean distance. The phase of each entry of $\mathrm{F}_{\mathrm{RF}}$ can thus be written as:

$\hat{\varphi}=(2 \pi \hat{n}) /\left(2^{B}\right)$ where, $\hat{n}$ is chosen according to

$$
\hat{\mathrm{n}}=\arg _{\mathrm{n} \in\left\{0, \ldots 2^{\mathrm{B}-1}\right\}} \min \left|\varphi-\frac{2 \pi \mathrm{n}}{2^{\mathrm{B}}}\right|
$$

where, $\varphi$ is the unquantized phase obtained from Equation (10). Then the unconstrained RF precoder $F_{R F}$ is computed through substituting quantized phase $\hat{\varphi}$ in Equation (11).

The optimal unconstrained precoder $\mathrm{F}^{*}$ can be written in terms of unconstrained RF precoder FRF and the uncontained baseband precoder $\mathrm{F}_{\mathrm{BB}}$ as:

$$
\mathrm{F}^{*}=\mathrm{F}_{\mathrm{RF}} \mathrm{F}_{\mathrm{BB}}
$$

From equation (12), we can write,

$$
\mathrm{F}_{\mathrm{BB}}=\left(\mathrm{F}_{\mathrm{RF}}^{\mathrm{T}} \mathrm{F}_{\mathrm{RF}}\right)^{-1} \mathrm{~F}_{\mathrm{RF}}^{\mathrm{T}} \mathrm{F}^{*}
$$

where, $F_{R F}^{T}$ is conjugate transformed form of $F_{R F}$

With consideration of four RF chains, the constrained analog $\mathrm{RF}$ precoder $\mathrm{F}_{\mathrm{RF}}$ and the constrained baseband precoder $\mathrm{F}_{\mathrm{BB}}$ are estimated using the following relation based on Conjugate Gradient square method:

$$
\begin{aligned}
& \min \left\|F^{*}-F_{R F} F_{B B}\right\|_{F} \\
& F_{R F}, F_{B B}
\end{aligned}
$$

$$
\text { s.t. }\left\|\mathrm{F}_{\mathrm{RF}} \mathrm{F}_{\mathrm{BB}}\right\|^{2} \mathrm{~F}=4
$$

Equation (12) can be written in modified form as:

$$
\mathrm{F}_{\mathrm{RF}}^{\mathrm{T}} \mathrm{F}_{\mathrm{RF}} \mathrm{F}_{\mathrm{BB}}=\mathrm{F}_{\mathrm{RF}}^{\mathrm{T}} \mathrm{F}^{*}
$$

In Equation (15), the unknown $\mathrm{F}_{\mathrm{BB}}$ can be determined iteratively with minimization of residual $r^{(i)}=F_{R F}{ }^{T} F^{*}-$ $\mathrm{F}_{\mathrm{RF}}{ }^{\mathrm{T}} \mathrm{F}_{\mathrm{RF}} \mathrm{F}_{\mathrm{BB}}{ }^{(\mathrm{i})}$ using Conjugate Gradient square method [13]. The iteration terminates when the estimated residual value is $\leq 1 \times 10^{-10}$.

The iteratively re estimated $\mathrm{F}_{\mathrm{BB}}$ value $\left(\mathrm{F}_{\mathrm{BB}}\right)$ is substituted in equation (8) to get

$$
\mathrm{F}^{*}=\mathrm{F}_{\mathrm{RF}} \mathrm{F}_{\mathrm{BB}}
$$

Equation (16) can be written in modified form as:

$$
\mathrm{F}_{\mathrm{BB}}{ }^{\mathrm{T}} \mathrm{F}_{\mathrm{RF}}=\mathrm{F}^{* \mathrm{~T}}
$$

where, $\mathrm{F}_{\mathrm{BB}}^{\mathrm{T}}$ and $\mathrm{F}^{* \mathrm{~T}}$ are conjugate transformed form of $\mathrm{F}_{\mathrm{BB}}$ 
and $\mathrm{F}^{*}$ respectively.

In Equation (17), the unknown $\mathrm{F}_{\mathrm{RF}}$ can be determined iteratively with minimization of residual $r^{(i)}=F^{*}-F_{B B}{ }^{T} F_{R F}{ }^{(i)}$ using Conjugate Gradient square method [13]. The iteration terminates when the estimated residual value is $\leq 1 \times 10^{-10}$.

The iteratively re estimated $F_{R F}$ value $\left(F_{R F}\right)$ would be such that

$$
\left\|F^{*}-\mathbf{F}_{\mathbf{R F}} \mathbf{F}_{\mathbf{B B}}\right\|_{F}=0 \text { and }\left\|\mathbf{F}_{\mathbf{R F}} \mathbf{F}_{\mathbf{B B}}\right\|_{F}^{2}=4
$$

In case of unconstrained $\mathrm{RF}$ combiner $\mathrm{W}_{\mathrm{RF}}$ at the receiver side, its each $(i, j)$ th element is given by

$$
\mathrm{W}_{\mathrm{RF}}(\mathrm{i}, \mathrm{j})=\frac{1}{\sqrt{\mathrm{N}_{\mathrm{r}}}} \mathrm{e}^{\mathrm{j} \varphi_{\mathrm{i}, \mathrm{j}}}
$$

Each entry of $\mathrm{W}_{\mathrm{RF}}$ are quantized up to $\mathrm{B}$ bits of precision, each quantized to its nearest neighbor based on closest Euclidean distance. The phase of each entry of $\mathrm{W}_{\mathrm{RF}}$ is estimated using equation (10). The unconstrained RF combiner $\mathrm{W}_{\mathrm{RF}}$ is computed through substituting quantized phase $\hat{\varphi}$ in Equation (19).

The optimal unconstrained combiner $\mathrm{W}^{*}$ can be written in terms of unconstrained RF combiner WRF and the uncontained baseband combiner $\mathrm{W}_{\mathrm{BB}}$ as:

$$
\mathrm{W}^{*}=\mathrm{W}_{\mathrm{RF}} \mathrm{W}_{\mathrm{BB}}
$$

From equation (20), we can write,

$$
\mathrm{W}_{\mathrm{BB}}=\left(\mathrm{W}_{\mathrm{RF}}^{\mathrm{T}} \mathrm{W}_{\mathrm{RF}}\right)^{-1} \mathrm{~W}_{\mathrm{RF}}^{\mathrm{T}} \mathrm{W}^{*}
$$

where, $\mathrm{W}_{\mathrm{RF}}^{\mathrm{T}}$ is conjugate transformed form of $\mathrm{W}_{\mathrm{RF}}$ [12]

With consideration of four RF chains, the constrained analog $\mathrm{RF}$ combiner $\mathrm{W}_{\mathrm{RF}}$ and the constrained baseband combiner $\mathrm{W}_{\mathrm{BB}}$ are estimated using the following relation based on Conjugate Gradient square method:

$$
\begin{aligned}
& \min \left\|\mathrm{W}^{*}-\mathrm{W}_{\mathrm{RF}} \mathrm{W}_{\mathrm{BB}}\right\|_{\mathrm{F}} \\
& \mathrm{W}_{\mathrm{RF}}, \mathrm{W}_{\mathrm{BB}} \\
& \text { s.t. }\left\|\mathrm{W}_{\mathrm{RF}} \mathrm{W}_{\mathrm{BB}}\right\|_{\mathrm{F}}^{2}=4
\end{aligned}
$$

Equation (20) can be written in modified form as:

$$
\mathrm{W}_{\mathrm{RF}}^{\mathrm{T}} \mathrm{W}_{\mathrm{RF}} \mathrm{W}_{\mathrm{BB}}=\mathrm{W}_{\mathrm{RF}}^{\mathrm{T}} \mathrm{W}^{*}
$$

In Equation (23), the unknown $\mathrm{W}_{\mathrm{BB}}$ can be determined iteratively with minimization of residual $r^{(i)}=W_{R F}^{T} W^{*}$. $\mathrm{W}_{\mathrm{RF}}^{\mathrm{T}} \mathrm{W}_{\mathrm{RF}} \mathrm{W}_{\mathrm{BB}}{ }^{(\mathrm{i})}$ using Conjugate Gradient square method [13]. The iteration terminates for the estimated residual valueis $\leq 1 \times 10^{-10}$.

The iteratively re estimated $\mathrm{W}_{\mathrm{BB}}$ value $\left(\mathrm{W}_{\mathrm{BB}}\right)$ is substituted in equation (20) to get

$$
\mathrm{W}^{*}=\mathrm{W}_{\mathrm{RF}} \mathrm{W}_{\mathrm{BB}}
$$

Equation (24) can be written in modified form as:

$$
\mathrm{W}_{\mathrm{BB}}^{\mathrm{T}} \mathrm{W}_{\mathrm{RF}}=\mathrm{W}^{* \mathrm{~T}}
$$

where, $\mathrm{W}_{\mathrm{BB}}^{\mathrm{T}}$ and $\mathrm{W}^{* \mathrm{~T}}$ are conjugate transformed form of $\mathrm{W}_{\mathrm{BB}}$ and $\mathrm{W}^{*}$ respectively.

In Equation (25), the unknown $\mathrm{W}_{\mathrm{RF}}$ can be determined iteratively with minimization of residual $\mathrm{r}^{(\mathrm{i})}=\mathrm{W}^{*} \mathrm{~T}-\mathrm{W}_{\mathrm{BB}}{ }^{\mathrm{T}} \mathrm{W}_{\mathrm{RF}}{ }^{(\mathrm{i})}$ using Conjugate Gradient square method [13]. The iteration terminates when the estimated residual value is $\leq 1 \times 10^{-10}$.

The iteratively re estimated $\mathrm{W}_{\mathrm{RF}}$ value $\left(\mathrm{W}_{\mathrm{RF}}\right)$ would be such that

$$
\left\|\mathrm{W}^{*}-\mathrm{W}_{\mathrm{RF}} \mathrm{W}_{\mathrm{BB}}\right\|_{\mathrm{F}}=0 \text { and }\left\|\mathrm{W}_{\mathrm{RF}} \mathrm{W}_{\mathrm{BB}}\right\|_{\mathrm{F}}^{2}=4
$$

In Appendix, our developed program for verifying $\left\|\mathrm{W}^{*}-\mathrm{W}_{\mathrm{RF}} \mathrm{W}_{\mathrm{BB}}\right\|_{\mathrm{F}}=0$ and $\left\|\mathrm{F}^{*}-\mathrm{F}_{\mathrm{RF}} \mathrm{F}_{\mathrm{BB}}\right\|_{\mathrm{F}}=0$ has been presented for developing idea for a typical assumed MIMO Rayleigh fading channel.

\section{System Description}

A simplified form of hybrid precoded millimetre wave wireless communication system is depicted in Figure 1. We consider that a color image of resolution 96 pixels (width) $\times 96$ pixels (height) will be processed in our simulated hybrid precoded millimeter wave wireless communication system.

The typically assumed color image is converted into three red, green and blue components with each component is of 96 pixels(width) $\times 96$ pixels(height). The pixel integer values [0-255] are converted into 8 bits binary form. The binary converted signal vector $S \in(0,1)$ of dimention $1 \times 221184$ is channel encoded and subsequently interleaved to produce a signal vector $\tilde{\mathrm{s}}$. The transformed signal vector $\tilde{\mathrm{s}}$ is of size $1 \times$ 442368. In case of merely $\mathrm{BCH}$ channel coding, the signal vector $\tilde{\mathrm{s}}$ would be of size1× 438912 and after 16arrayQAM/PSK/DPSK digital modulation [14], the number of digitally modulated symbols is 109728 and on adding additional 864 zeros in zero padding scheme. However, $110592 \times 1$ sized digitally modulated signal vector $\widehat{\mathrm{s}}$ is processed in serial to paralel converter to produce blocks with each block containing $\mathrm{N}(=1024)$ number of digitally modulated complex symbols $\left[\hat{\mathrm{X}}_{0}, \hat{\mathrm{X}}_{1}, \hat{\mathrm{X}}_{2} \ldots \ldots \ldots . . \hat{\mathrm{X}}_{\mathrm{N}-1}\right]$ prior to 1024 point IDFT implementation in OFDM modulation [10]. The number of OFDM block is 108. In each OFDM block, the samples are represented by $\left[\hat{\mathrm{X}}_{0}, \hat{\mathrm{X}}_{1}, \hat{\mathrm{X}}_{2} \ldots \ldots \ldots . . \hat{\mathrm{X}}_{\mathrm{N}-1}\right]$ and we can write,

$$
\begin{array}{r}
\hat{X}_{k}=\frac{1}{N} \sum_{n=0}^{n=N-1} \widehat{X}_{n} e^{j 2 \pi n k / N} \\
\text { for } k=0,1,2,3, \ldots \ldots N-1
\end{array}
$$

The IDFT implemented $1024 \times 108$ sized data vector $\dddot{X}$ is reshaped into single column data vector $\overrightarrow{\mathrm{X}}$ of dimension $110592 \times 1$ in parallel to serial converter and passed through Spatial demultiplexer to produce 4 data stream of a $4 \times 27648$ sized signal vector $X$. The signal vector $X$ is multiplied by a digital baseband precoder matrix FBB of dimension $4 \times 4$ and the digitally precoded signal is undergone in $\mathrm{D} / \mathrm{A}$ conversion 
with execution of up sampling and filtering with raised cosine pulse shaping digital filter [15]. The D/A converted filtered $4 \times 110632$ sized signal vector DA is multiplied with $256 \mathrm{x}$ 4sizedanalog RF precoder FRF to produce $256 \mathrm{x}$ 110632 sized signal vector FR. In RF up converter section, the signal is multiplied for each of 256 channels with multiplier $\quad \mathrm{ML}=\exp (1 \mathrm{i} * 2 *$ pi*Carrier_Freq. $* t)$, where, Carrier_Freq is the carrier frequency in mmWave band
$(38 \mathrm{GHz})$ and $\mathrm{t}$ is the sample time for each of the sample ranging from 1 to 110632 . A 256 x 110632 sized matrix MLL can be generated from ML using MATLAB notation MLL= repmat (ML, 256,1); The transmitted signal TX is given by $\mathrm{TX}=\mathrm{FR} \bullet \mathrm{MLL}$, where, $\bullet$ is the hadamard product which is indicative of element wise multiplication of two matrices.
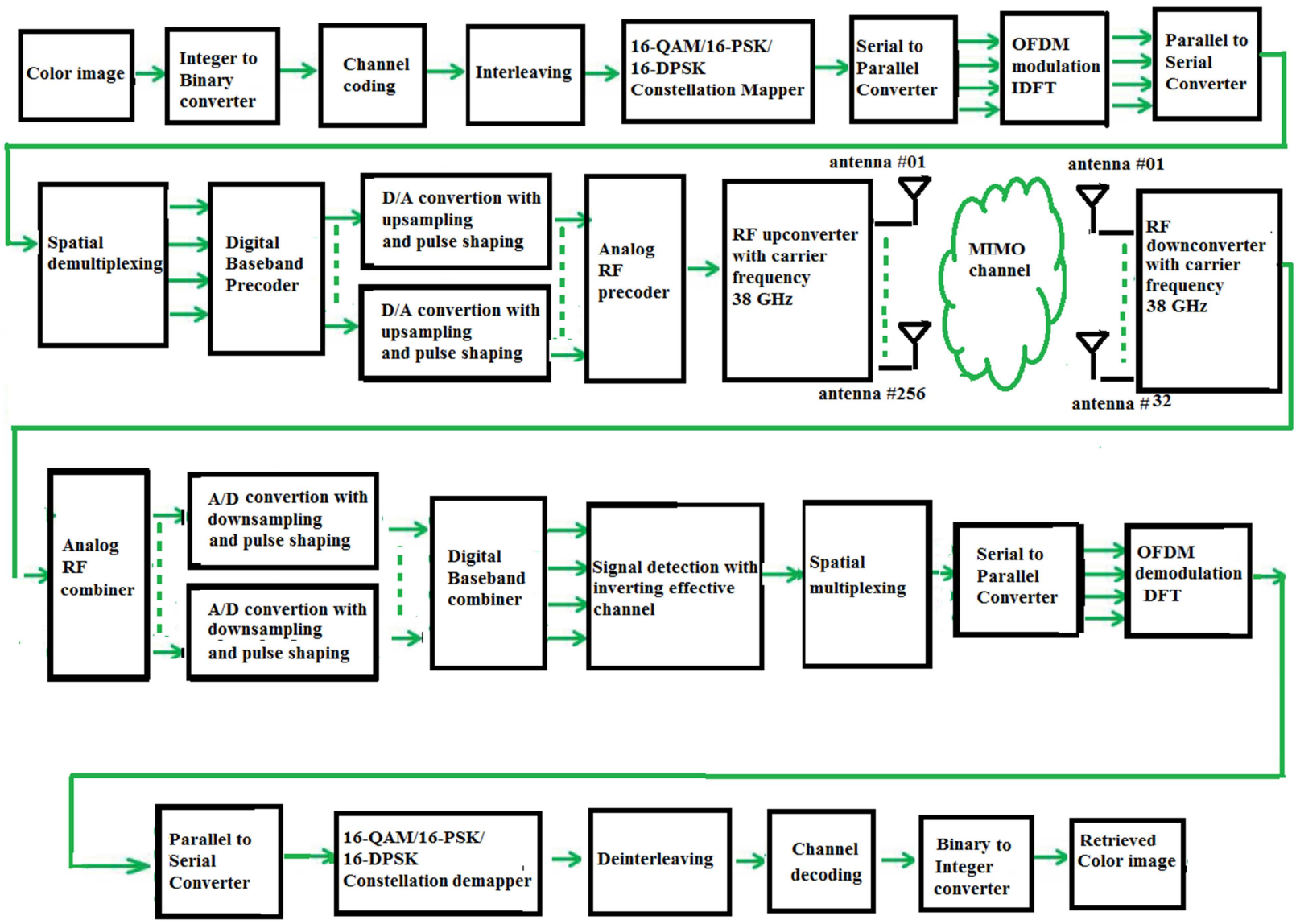

Figure 1. Block diagram of Hybrid Precoded Millimeter Wave Wireless communication system.

In receiving section, the 32x 110632 sized received signal $\mathrm{Y}$ with consideration of MIMO fading channel $\mathrm{H}$ is given by:

$$
\mathrm{Y}=\mathrm{HTX}
$$

The received signal is RF down converted through multiplication of $32 \times 110632$ sized matrix MLLL. The matrix MLLL can be generated from a matrix MLP using MATLAB notation

MLLL $=\quad \operatorname{repmat}(\mathrm{MLP}, \quad 32,1) \quad$ where, $\quad \mathrm{MLP}=\exp (-$ $1 i^{*} 2 *$ pi*Carrier_Freq. $\left.* t\right)$. The received signal $Y$ after multiplication with MLLL and in presence of addictive complex Gaussian noise $\mathrm{N}$ with i.i.d. $\mathrm{CN}\left(0, \sigma^{2}\right)$ is given by

$$
\tilde{\mathrm{Y}}=\mathrm{Y} \bullet \mathrm{MLLL}+\mathrm{N}=\mathrm{HFRFDA}+\mathrm{N}
$$

The noisy received signal is passed through $32 \times 4$ sized analog RF combiner WRF to produce modified form of received signal $\mathrm{Y}$ as:

$$
\overline{\mathrm{Y}}=\mathrm{WRF}^{\mathrm{T}} \tilde{\mathrm{Y}}=\mathrm{WRF}^{\mathrm{T}} \mathrm{HFRFDA}+\mathrm{WRF}^{\mathrm{T}} \mathrm{N}
$$

On executing A/D conversion theA/D converted $4 \times 27648$ sized received signal vector $\hat{\bar{Y}}$ is given by

$$
\hat{\overline{\mathrm{Y}}}=\mathrm{WRF}^{\mathrm{T}} \operatorname{HFRFFBB}^{\mathrm{X}}+\mathrm{WRF}^{\mathrm{T}} \overline{\mathrm{N}}
$$

After passing through $4 \times 4$ digital baseband combiner WBB, we can write,

$$
\vec{Y}=W B B^{T} \hat{\bar{Y}}=W B B^{T} W R F^{T} \operatorname{HFRFFBB} \overline{\mathrm{X}}+W B B^{T} W R F^{T} \bar{N}
$$

Where, $\mathrm{WBB}^{\mathrm{T}}$ is the complex conjugate transformed form of WBB.

In Equation 32, it is quite observable that the effective MIMO channel $\overline{\mathrm{H}}$ is given by

$$
\overline{\mathrm{H}}=\mathrm{WBB}^{\mathrm{T}} \mathrm{WRF}^{\mathrm{T}} \mathrm{HFRFFBB}
$$

On applicability of ZF based signal detection technique, the spatially demultiplexed signal $\overline{\mathrm{X}}$ is detected. The detected signal is spatially multiplexed to produce a single column 
data vector $\overrightarrow{\vec{X}}$ of dimension $110592 \times 1$. In serial to parallel converter, the signal vector $\overrightarrow{\vec{X}}$ is reshaped into $1024 \times 108$ sized data vector $¥$. Ineach of 108 blocksof $¥$, the samples are represented by $\left[\mathrm{K}_{0}, \mathrm{~K}_{1}, \mathrm{X}_{2} \ldots \ldots \ldots \ldots \mathrm{K}_{\mathrm{N}-1}\right]$ and after implementation of 1024 point DFT in OFDM demodulation section, the samples in each block is represented by

$$
\bar{X}_{k}=\sum_{n=0}^{n=N-1} X_{n} e^{-j 2 \pi n k / N}
$$

$$
\text { for } \mathrm{k}=0,1,2,3, \ldots \ldots \ldots \mathrm{N}-1
$$

The DFT implemented $1024 \times 108$ sized data vector $\overline{\mathrm{X}}$ is processed for parallel to serial conversion, digitally demodulation, de interleaving, channel decoding, binary to integer conversion to retrieve eventually the transmitted image.

\section{Results and Discussion}

We have conducted computer simulation study using MATLAB R2014a to evaluate the quality of the transmitted color image in a Hybrid Precoded Millimeter Wave Wireless Communication System based on the parameters presented in Table $1 \mathrm{It}$ is assumed that the channel state information (CSI) of the mmWave MIMO fading channel is available at the receiver and the fading process is approximately constant during the whole period of color I mage transmission.

On critical observation of grapical illustrations presented in Figure 2 through Figure 4, it is found that the performance of the simulated systemis very much well definedunder the considered simulation parameters. In all cases, the system shows satisfactory performance in 16-QAM digital modulation and comparitively worst performancein 16DPSKdigital modulation. In Figure 2 with consideration of $1 / 2$ -rated convolutional channel coding, the ber values at $1 \mathrm{~dB}$ SNRarefound to have values of $0.1514,0.2182$ and 0.4733 in case of 16-QAM, 16-PSK and 16-DPSK which is in dicative of system performance improvement of1.59 dBin16-QAM as compared to16-PSK and $4.95 \mathrm{~dB}$ in 16-QAM as compared to 16-DPSK.At $10 \%$ BER, achieved system performance improvement in terms of signal to noise ratio (SNR) are 0.5 $\mathrm{dB}$ and $4.8 \mathrm{~dB}$ in 16-QAM relative to 16-PSK and16-DPSK respectively. In Figure 3 for LDPC channel coding, the ber values at $1 \mathrm{~dB}$ SNRare $0.0457,0.1879$ and 0.3526 for 16 QAM, 16-PSK and 16-DPSK respectively which implies system performance improvement of $6.14 \mathrm{~dB}$ in 16 QAMrelative to 16-PSK and $8.87 \mathrm{~dB}$ in16-QAM relative to16-DPSK. In Figure 4 for $\mathrm{R}$ and $\mathrm{A}$ channel coding, the system performance in all considered digital modulations shows almost linear response viz. improvement of system performance occurs linearly with increase in SNR values. At a typically assumed SNR value of $1 \mathrm{~dB}$, the ber valuesare $0.1705,0.2186$ and 0.2472 for 16-QAM, 16-PSK and 16DPSK respectively which implies system performance improvement of $1.08 \mathrm{~dB}$ in 16-QAMrelative to 16-PSK and $1.61 \mathrm{~dB}$ in 16-QAMrelative to16-DPSK. In Figure 5 for $\mathrm{BCH}$ channel coding, the estimated ber valuesat SNR value of 1 $\mathrm{dB}$ are $0.1278,0.1809$ and 0.2583 for $16-\mathrm{QAM}, 16-\mathrm{PSK}$ and 16-DPSK respectively which is indicative of system performance improvement of $1.51 \mathrm{~dB}$ in 16-QAMrelative to16-PSKand $3.06 \mathrm{~dB}$ in 16-QAMrelative to 16-DPSK. In Figure 6, it is observable that the simulated system shows satisfactory performance in retrieving color image. At reasonably low SNR value, the quality of the retrieved color image is acceptable.

\begin{tabular}{|c|c|}
\hline Parameters & Types \\
\hline Data type: Color image & 96 pixels(height) $\times 96$ pixels(width) \\
\hline Antenna configuration & $256($ Transmitting $) \times 32($ Receiving $)$ \\
\hline Digital modulation & 16-PSK and 16-QAM and 16-DPSK \\
\hline Channel coding & $\begin{array}{l}\text { LDPC, } \mathrm{R} \text { and } \mathrm{A} \text {, Convolutional and } \\
\mathrm{BCH}\end{array}$ \\
\hline LDPC decoding Algorithm & Log Domain Sum-Product \\
\hline OFDM Block Size & 1024 digitally modulated symbols \\
\hline OFDM symbol duration(sec) & $5.1200 x^{-05}$ \\
\hline Upsampling frequency $(\mathrm{Hz})$ & 80000000 \\
\hline Orthogonal subcarrier spacing $(\mathrm{Hz})$ & $1.9531 \times 10^{04}$ \\
\hline System bandwidth (MHz) & 20 \\
\hline $\begin{array}{l}\text { Sampling time for transmitted } \\
\text { signal(sec) }\end{array}$ & $1.2500 \times 10^{-8}$ \\
\hline Oversampling rate & 4 \\
\hline Pulse shaping digital filter & Square root raised cosine filter \\
\hline Order of filter & 40 \\
\hline Roll of Factor of filter & 0.25 \\
\hline Filter delay(\# of input samples) & 5 \\
\hline Carrier frequency $(\mathrm{GHz})$ & 28 \\
\hline $\begin{array}{l}\text { Path loss model }(\mathrm{dB}) \\
\lambda=\text { wavelength }(\mathrm{m}) \text { of carrier } \\
\text { frequency, } \mathrm{d}=\text { distance }(\mathrm{m}) \text { between } \\
\text { transmitter and receiver }\end{array}$ & $-20 \log _{10}(\lambda /(4 \pi d)$ \\
\hline $\begin{array}{l}\text { No. of RF chains in both } \\
\text { Transmitter and Receiver sides }\end{array}$ & 4 \\
\hline Number of channel paths & 6 \\
\hline Size of Baseband Precoder $F_{\mathrm{BB}}$ & $4 \times 4$ \\
\hline Size of RF Precoder $F_{R F}$ & $256 \times 4$ \\
\hline Size Baseband Combiner $\mathrm{W}_{\mathrm{BB}}$ & $4 \times 4$ \\
\hline Size RF Combiner $\mathrm{W}_{\mathrm{RF}}$ & $32 \times 4$ \\
\hline SNR & $0-10 \mathrm{~dB}$ \\
\hline Signal detection techniques used & Zero forcing $(\mathrm{ZF})$ \\
\hline Channel & $\begin{array}{l}\text { AWGN and Ray path based } \\
\text { geometrical MIMO }\end{array}$ \\
\hline
\end{tabular}

Table 1. Summary of simulation model parameters.

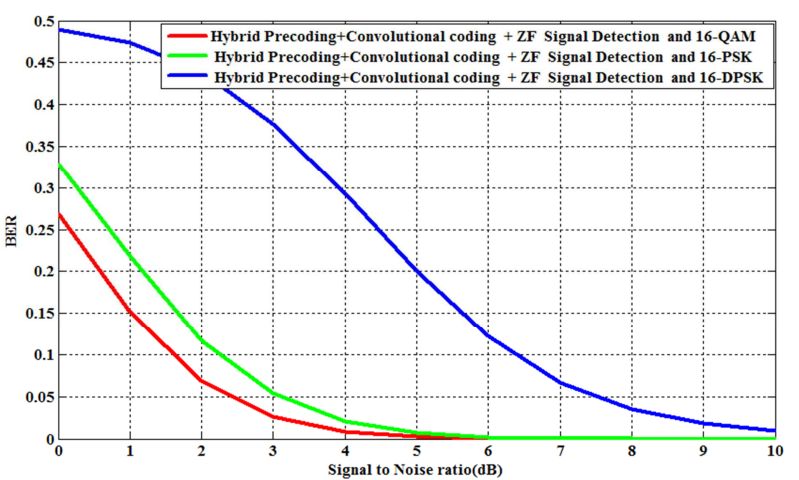

Figure 2. BER performance comparison of Hybrid Precoded Millimeter Wave Wireless Communication system with implementation of ZF signal detection, $1 / 2$-rated convolutional channel coding and various digital modulation schemes. 


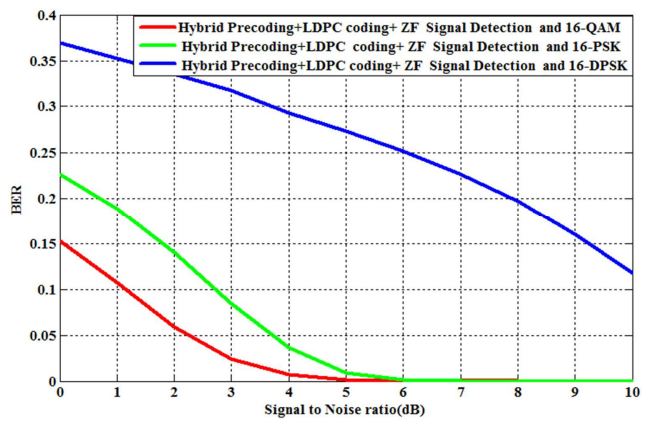

Figure 3. BER performance comparison of HybridPrecoded Millimeter Wave Wireless Communication system with implementation of $Z F$ signal detection, LDPC channel coding and various digital modulation schemes.

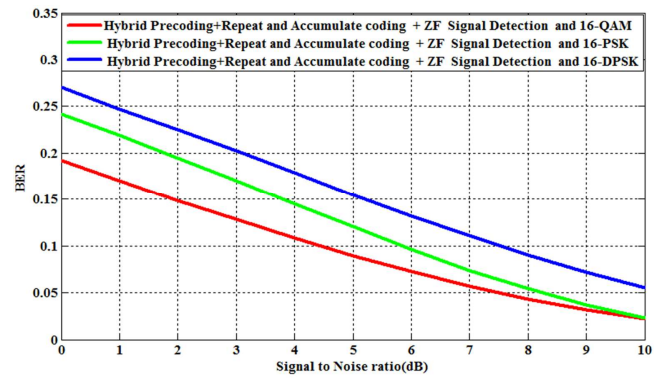

Figure 4. BER performance comparison of Hybrid Precoded Millimeter Wave Wireless Communication system with implementation of $Z F$ signal detection, Repeat and Accumulate channel coding and various digital modulation schemes.

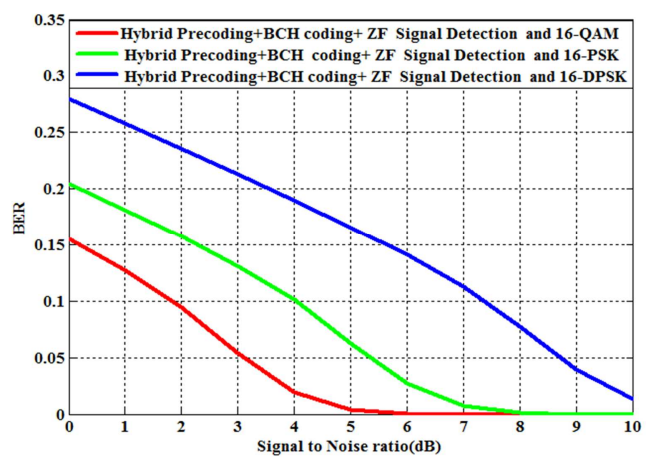

Figure 5. BER performance comparison of Hybrid Precoded Millimeter Wave Wireless Communication system with implementation of $Z F$ signal detection, BCH channel coding and various digital modulation schemes.

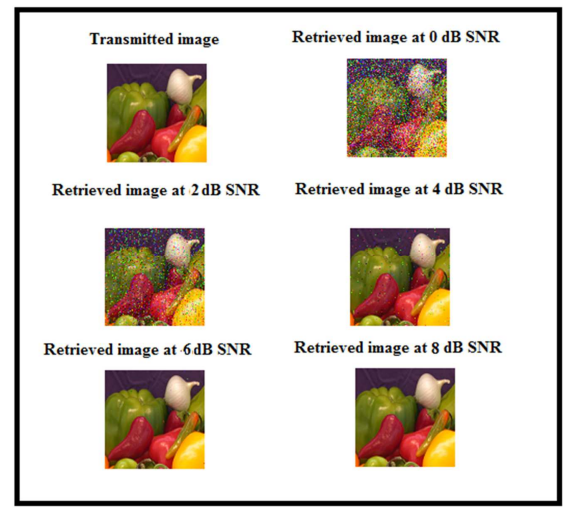

Figure 6. Transmitted and retrieved images in Hybrid Precoded Millimeter Wave Wireless Communication system under implementation of $Z F$ signal detection, LDPC channel coding and 16-QAM digital modulation scheme.

\section{Conclusions}

In this present paper, we have made a comprehensive study on the performance analysis of millimeter wave (mmWave) wireless communication system under simultaneous implementation of both digital and analog precoding and combining schemes in hybrid form. Simulation results ratify that the Hybrid precoders and combiners have been designed satisfactorily with the application of effective iterative Conjugate Gradient Squared (CGS) Method and Singular value decomposition of ray path geometry based mmWave MIMO fading channel. Based on the results presented in this paper on color image transmission, it can be concluded that the presently considered Hybrid Precoded Millimeter Wave wireless communication System is undoubtedly a robust system in perspective of signal transmission in hostile fading channel under implementation of LDPC channel coding, 16 QAM digital modulation and Zero Forcing (ZF) signal detection schemes.

\section{Appendix}

clear all; close all;

\%antenna configuration: 32 receiving $\times 256$ transmitting

$\%$ MIMO fading channel generation

$\mathrm{H}=\operatorname{sqrt}(1 / 2) *(\operatorname{randn}(32,256)+\operatorname{sqrt}(-1) * \operatorname{randn}(32,256))$;

$\%$ MIMO channel

$\%$ Normalization of channel matrix

for $\mathrm{kk}=1: 256$

for $\mathrm{kkk}=1: 32$

$\mathrm{H}(\mathrm{kkk}, \mathrm{kk})=\mathrm{H}(\mathrm{kkk}, \mathrm{kk}) /(\operatorname{abs}(\mathrm{H}(\mathrm{kkk}, \mathrm{kk})))$;

end; end;

channel_normalization $=\left(\right.$ norm $\left.\left(\mathrm{H}, \mathrm{fro}^{\prime}\right)\right) .^{\wedge} 2$;

$\%$ its value would be $32 \times 256=8192$

\%\%\%\%\%\%\%\%\%\%\%\%\%\%\%\%\%\%\%\%\%\%\%\%\%\%\%\%\%

$\%$ Singular value decomposition (SVD) of channel matrix $\mathrm{H}$

[U SIGMA VT] $=\operatorname{svd}(\mathrm{H}) ; \% \mathrm{U:} 32 \times 32$

$\mathrm{V}=\mathrm{VT}^{\prime} ; \% 256 \times 256$

FSTAR $=\mathrm{V}(:, 1: 4) ; \% 256 \times 4$, optimal unconstrained precoder WSTAR $=\mathrm{U}(:, 1: 4) ; \% 256 \times 4$ optimal unconstrained combiner

$\%$ FSTAR $=$ Baseband Precoder(FBB) X RF Precoder(FRF)

$\%$ WSTAR=Baseband Combiner(WBB) X RF

Combiner(WRF)

\%\%\%\%\%\%\%\%\%\%\%\%\%\%\%\%\%\%\%\%\%\%\%\%\%\%\%\%\% $\% \%$

$\%$ Unconstrained FRF and FBB estimation

$\%$ no of stream $=4, F R F=256 \times 4$

quantized_phase $=\left(\left(0: 2^{\wedge} 7-1\right) * 2 * \text { pi } / 2^{\wedge} 7\right)^{\prime}$;

unquantized_phase $=2 *$ pi $*$ rand $(256,4) ; \% 256$ rows x 4 cols

for $\mathrm{kk}=1: 4$

for $\mathrm{kkk}=1: 256$

for $\mathrm{kkkk}=1: 128$

nhat $(\mathrm{kkk}, \mathrm{kk}, \mathrm{kkkk})=$ abs $(($ unquantized_phase $(\mathrm{kkk}, \mathrm{kk})$ -

quantized_phase(kkkk)));

end; end; end;

for $\mathrm{kk}=1: 4$ 
for $\mathrm{kkk}=1: 256$

[value $(\mathrm{kkk}, \mathrm{kk})$, integer(kkk,kk)]= $\min (\operatorname{nhat}(\mathrm{kkk}, \mathrm{kk},:))$; phihat $(\mathrm{kkk}, \mathrm{kk})=2 *$ pi*integer $(\mathrm{kkk}, \mathrm{kk}) /\left(2^{\wedge} 7\right)$;

end; end;

$\% \% \% \% \% \% \% \% \% \% \% \% \% \% \% \% \% \% \% \%$

$\mathrm{FRF}=(1 / \operatorname{sqrt}(256)) * \exp (\mathrm{j} *$ phihat $) ; \% 256 \times 4 \mathrm{RF}$ Precoder

$\% \% \% \% \% \% \% \% \% \% \% \% \% \% \% \% \% \% \% \% \% \% \% \% \%$

$\mathrm{FBB}=\mathrm{inv}\left(\mathrm{FRF}{ }^{*} \mathrm{FRF}\right) * \mathrm{FRF}^{*} \mathrm{FSTAR}$

$\% \% \% \% \% \% \% \% \% \% \% \% \% \% \% \% \% \% \% \% \% \% \% \% \%$

$\%$ Reestimate FRF and FBB such that FRF*FBB=FSTAR

modified_FRF $=$ FRF $*$ FRF;

modified_FSTAR $=$ FRF $^{*}$ FSTAR;

$\%$ Conjugate Gradient square Method

for $\mathrm{kk}=1: 4$

Estimated_FBB $(:, \mathrm{kk})=$

cgs(modified_FRF,modified_FSTAR(:,kk), 1e-10);

end;

$\% \mathrm{FBB}^{\prime} * \mathrm{FRF}^{\prime}=\mathrm{FSTAR}^{\prime}, \mathrm{FSTAR}^{\prime}=8$ rows $\mathrm{x} 256$ cols $\mathrm{FRF}^{\prime}=$

8 rows $\times 256$ cols

FSTART $=$ FSTAR';

FBBT $=$ Estimated FBB';

for $\mathrm{kk}=1: 256$

Estimated_FRF(:,kk)= $\operatorname{cgs}(\operatorname{FBBT}, F S T A R T(:, k k), 1 \mathrm{e}-10)$; end;

Estimated_FRF=Estimated_FRF';

$\%$ Restimate WRF and WBB such that WRF*WBB=WSTAR unquantized phase $1=2 *$ pi* $\operatorname{rand}(32,4) ; \% 32$ rows $\times 8$ cols $\%$ From Low-Complexity Hybrid Precoding equation 6 , quantized phase

for $\mathrm{kk}=1: 4$

for $\mathrm{kkk}=1: 32$

for $\mathrm{kkkk}=1: 128$

nhat $1(\mathrm{kkk}, \mathrm{kk}, \mathrm{kkkk})=\mathrm{abs}(($ unquantized phase1 $(\mathrm{kkk}, \mathrm{kk})-$

quantized_phase(kkkk)));

end;end; end;

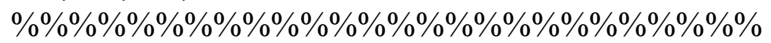

for $\mathrm{kk}=1: 4$

for $\mathrm{kkk}=1: 32$

[value1(kkk,kk), integer1 $(\mathrm{kkk}, \mathrm{kk})]=\min ($ nhat1 $(\mathrm{kkk}, \mathrm{kk},:))$; phihat $1(\mathrm{kkk}, \mathrm{kk})=2{ }^{*}$ pi ${ }^{*}$ integer $1(\mathrm{kkk}, \mathrm{kk}) /\left(2^{\wedge} 7\right)$;

end; end;

\%\% \% \% \% \% \% \% \% \% \% \% \% \% \% \% \% \% \% \% \%

$\mathrm{WRF}=(1 / \operatorname{sqrt}(32)) * \exp (\mathrm{j} *$ phihat 1$) ; \% 32 \times 4$ RF Precoder

$\mathrm{WBB}=\mathrm{inv}\left(\mathrm{WRF}^{\prime} * \mathrm{WRF}\right) * \mathrm{WRF}^{*} \mathrm{WSTAR}^{*}$

\%\% \% \% \% \% \% \% \% \% \% \% \% \% \% \% \% \% \% \% \% \% \% \% \% \% \% \% $\%$

modified $\mathrm{WRF}=\mathrm{WRF} * \mathrm{WRF}$;

modified ${ }^{-}$WSTAR $=W^{\prime} F^{*}$ WSTAR;

$\%$ Equation modified_WRF*WBB $=$ modified_WSTAR

$\%$ Conjugate Gradient square Method

for $\mathrm{kk}=1: 4$

Estimated_WBB $(:, \mathrm{kk})=$

cgs(modified_WRF,modified_WSTAR(:,kk), 1e-10);

end;

$\% \mathrm{WBB}^{\prime *} \mathrm{WRF}^{\prime}=\mathrm{WSTAR}^{\prime}, \mathrm{WSTAR}^{\prime}=8$ rows $\times 256$ cols

$\mathrm{WRF}^{\prime}=8$ rows $\times 256$ cols

WSTART $=$ WSTAR';
WBBT=Estimated_WBB';

for $\mathrm{kk}=1: 32$

Estimated_WRF(:,kk)= cgs(WBBT,WSTART(:,kk), 1e-10); end;

Estimated_WRF $=$ Estimated_WRF';

\% \% \% \% \% \% \% \% \% \% \% \% \% \% \% \% \% \% \% \% \% \% \% \% \% \% \% \% result $1=$ round(norm(FSTAR-

Estimated_FRF*Estimated_FBB,'fro'))

result $2=$ round $($ norm $($ WSTAR-

Estimated WRF*Estimated WBB,'fro'))

\% $\% \% \% \% \% \% \% \% \% \% \% \% \% \% \% \% \% \% \% \% \% \% \% \% \% \% \%$

\section{References}

[1] Sooyoung Hur, Sangkyu Baek, Byungchul Kim, Youngbin Chang, Andreas F. Molisch, Theodore S. Rappaport, Katsuyuki Haneda, and Jeongho Park, 2016: Proposal on Millimeter-Wave Channel Modeling for 5G Cellular System, Transactions on Wireless Communications, vol. PP, issue 99, pp. 1-16.

[2] Diana Maamari, Natasha Devroye, Daniela Tuninetti, 2016: Coverage in mmWave Cellular Networks with Base Station Cooperation, Transactions on Wireless Communications, vol. PP, issue 99, pp. 1-14.

[3] M. N. Kulkarni; A. Ghosh; J. G. Andrews, 2016:A Comparison of MIMO Techniques in Downlink Millimeter Wave Cellular Networks with Hybrid Beamforming, IEEE Transactions on Communications, vol. PP, issue 99, pp. 1-18.

[4] Jonathan Rodriguez, 2015: Fundamentals of 5G Mobile Networks, John Wiley and Sons, Ltd, United Kingdom.

[5] Peter Sweeney, 2002: Error Control Coding, From Theory to Practice John Wiley and Sons limited, England.

[6] Yuan Jiang, 2010: A Practical Guide to Error-Control Coding Using MATLAB, Artech House, Boston, USA.

[7] Bagawan Sewu Nugroho, https://sites.google.com/site/bsnugroho/ldpc.

[8] Giorgio M. Vitetta, Desmond P. Taylor, Giulio Colavolpe, Fabrizio Pancaldi andPhilippa A. Martin,2013:Wireless Communications Algorithmic Techniques.John Wiley and Sons Ltd, United Kingdom.

[9] Sneha Bawane and V. V. Gohokar, 2014: Simulation of Convolutional Encoder, International Journal of Research in Engineering and Technology (IJRET), India, 3(3), 557-561.

[10] Yong Soo Cho, Jackson Kim, Won Young Yang, Chung G. Kang, 2010: MIMO-OFDM Wireless Communications with MATLAB, John Wiley and Sons (Asia) PTE Limited, Singapore.

[11] Ahmed Alkhateeb, Geert Leus, and Robert W. Heath Jr., 2015: Limited Feedback Hybrid Precoding for Multi-User Millimeter Wave Systems, IEEE Transactions on Wireless Communications, vol. 14, issue 11, pp. 6481-6494.

[12] Weiheng Ni, Xiaodai Dong, and Wu-Sheng Lu, 2015: NearOptimal Hybrid Processing for Massive MIMO Systems via Matrix Decomposition, pp. 1-9, http://arxiv.org/abs/1504.03777. 
[13] Richard Barrett, Michael Berry, Tony F. Chan, James Demmel, J une M. Donato, Jack Dongarra, Victor Eijkhout, Roldan Pozo, Charles Romine and Henk Van der Vorst, 1994: Templates for the Solution of Linear Systems: Building Blocks for Iterative Methods, SIAM, Philadelphia, USA.

\section{Bibliography}

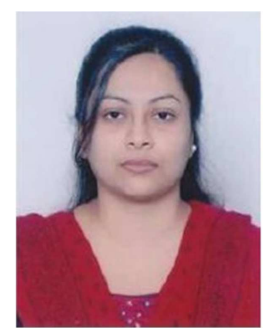

Shammi Farhana Islam is working as a lecturer in the Department of Materials Science and Engineering, Faculty of Engineering, University of Rajshahi, Bangladesh. She received her B.Sc. (Hons.) and M.Sc. degree both in Applied Physics and Electronic Engineering department from university of Rajshahi in 2009 and 2010 respectively. During her post graduate study, she has completed a Signal Processing based research work on EMG Signal Analysis Using Wavelet Transform. Her research interest is in Applicability of various digital signal processing techniques in Audio, text and Video (one dimensional, Stereographic and multiview) signal transmission in hostile channels and their effective retrieval.

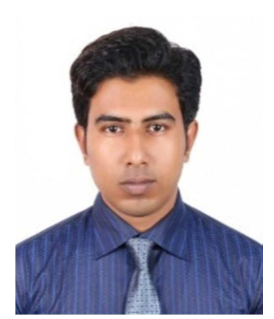

Sk. Shifatul Islam did his B.Sc(Hons) and M.Sc degree in Applied Physics and Electronic Engineering from University of Rajshahi in 2014 and 2015. Concurrently, he is engaged with simulation study on MIMO wireless communication and signal processing. His research interest is in Transmit/Receive antenna diversity, Channel coding, MIMO-OFDM, MCCDMA, DCT-IFDMA, MGSTC, Non Orthogonal Frequency Division Multiple Access (NOMA), mmWave system in Audio, text and Video (one dimensional and multiview) signal transmission in hostile channels and their effective retrieval.
[14] Theodore. S. Rappaport, 2004: Wireless communications: Principles and Practices, Second Edition, Prentice Hall Inc., New Jersey, USA.

[15] Mathuranathan Viswanathan, 2013: Simulation of Digital Communication systems using Matlab, second edition.

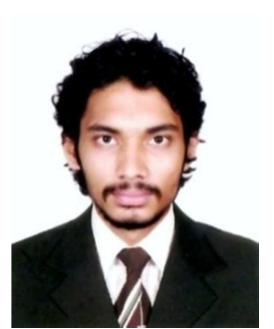

Mahmudul Haque Kafi received his B.Sc(Hons) and M.Sc degree in Applied Physics and Electronic Engineering from University of Rajshahi in 2014 and 2015 respectively. Concurrently, he is engaged with simulation study on MIMO wireless communication and signal processing. His research interest is in Transmit/Receive antenna diversity, Internet of things (IoT), Channel coding, MIMO-OFDM, MCCDMA, DCT-IFDMA, MGSTC, Non Orthogonal Frequency Division Multiple Access (NOMA), mmWave system in Audio, text and Video (one dimensional and multiview) signal transmission in hostile channels and their effective retrieval.

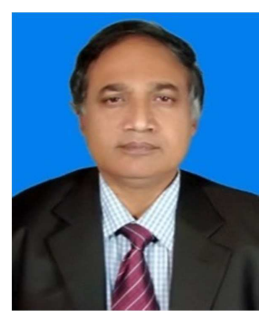

Shaikh Enayet Ullah is a Professor of the Department of Applied Physics and Electronic Engineering, University of Rajshahi, Bangladesh. He received US equivalent B.Sc and M.Sc degree in Physics and Electronics and Ph.D degree in Physics in 1983,1985 and 2000 respectively. $\mathrm{He}$ worked as a Professor and Chairman (on deputation) in the Department of Information and Communication Engineering, University of Rajshahi. He is working as a member of both Editorial and Reviewer Board of various International Journals. He has published more than 90 papers in multidisciplinary fields. His main research interests include $5 \mathrm{G}$ copatibale mmwave communications, Masssive MIMO communications, COMP in MIMO OFDM/OFDMA and Channel equialization. 